Sciensage

Journal of Advanced Scientific Research

Available online through https: / /sciensage.info
ISSN: 0976-9595

Research Article

DOI: $10.55218 /$ JASR. 202213124

\title{
PROFICIENT PHOTOCATALYTIC DEGRADATION OF METHYLENE BLUE DYE USING Ti-DOPED CdSe NANOPARTICLES UNDER SOLAR LIGHT IRRADIATION
}

\author{
R. Emelda ${ }^{1}$, M. Jaya Rajan ${ }^{1}$, P. Muthirulan* ${ }^{2}$ \\ ${ }^{1}$ Department of Chemistry, AnnaiVelankanni College, Tholayavattam, TamilNadu, India \\ ${ }^{2}$ Department of Chemistry, Lekshmipuram College Arts and Science, Neyyoor, TamilNadu, , India \\ (Affiliated to Manonmaniam Sundaranar University, Tirunelveli, TamilNadu, India) \\ *Corresponding author: pmuthirulan@gmail.com
}

\begin{abstract}
The present analysis explores the photocatalytic activity of Ti-doped CdSe and undoped CdSe nanoparticles towards Methylene Blue (MB) dye degradation under solar light irradiation. The as prepared $\mathrm{Ti}-\mathrm{CdSe}$ and mere $\mathrm{CdSe}$ nanoparticles were characterized using XRD, UV, FTIR, TGA, SEM and TEM analysis. TEM micrographs confirm the synthesized samples are in nanometer regime. The insertion of Titania onto CdSe lattice was confirmed by XRD and SEM-EDAX analysis. The absorption spectrum of Ti doped CdSe nanoparticles showed blue shift (lower wavelength) than mere CdSe. Ti-CdSe nanoparticles have greater thermal stability than mere CdSe nanoparticles. The photocatalytic activity of the Ti-CdSe nanoparticles photocatalyst was determined by degrading a MB dye under solar light irradiation. Ti-CdSe nanoparticles maintain high photocatalytic activity under solar light irradiation in comparison to mere CdSe nanoparticles. Low electron-hole pair recombination with increased efficiency was observed under solar light due to the strong absorption by the photocatalyst under solar light. The main active species responsible for catalytic degradation are $\mathrm{H}^{+}$and $\mathrm{OH}^{\cdot}$. The probable catalytic mechanism was proposed from the experimentally derived results.
\end{abstract}

Keywords: Ti-CdSe nanoparticles, Methylene blue dye, Solar light, Photodegradation.

\section{INTRODUCTION}

Today mankind is facing major problems in environmental deterioration, because of organic pollutants contaminating air, water and soil [1-3]. Industrial waste, toxic gases, pesticides, fertilizers, heavy metals are important contaminants for deteriorating the environment [4]. Textile industries are using 10-12\% dyestuffs such as Rose Bengal, Thymol Blue, Methylene blue, Indigo Carmine, Rhodamine $\mathrm{B}$ etc which contaminates fresh water bodies such as rivers and lakes $[5,6]$. These dyestuffs and hazardous materials cause illness on human beings and also aquatic species. Various methods had been used for decades such as adsorption, coagulation, flocculation, reverse osmosis, photocatalysis etc. to get rid of the organic pollutant from wastewater discharged from these factories [7]. Among these methods, photocatalysis is the effective and low cost highly active methods for the removal of dyestuffs from the industrial waste water as well as the reaction that takes place by the inexhaustible solar energy [8].

Nanotechnology is broadening its wide angles towards removal of the dyestuffs using the peculiar characteristics of nanoscale materials. The recent analysis is focusing on the removal of organic pollutants by using semiconducting nanomaterials that act as photocatalyst [9]. This leads to the absorption of photons in a huge surface area than the band gap by two types of semiconducting materials like homogeneous and heterogeneous. The photodegradation efficiency is enhanced by using heterogeneous semiconducting materials; the various strategies are involved by increasing activity of photocatalyst such as doping, band gap, size and morphology $[10,11]$.

CdSe is one of the important semiconducting materials due to highly stable, low cost and effective oxidation materials. The band gap energy of CdSe nanoparticles is $1.6-1.8 \mathrm{eV}$ [12]. In a photocatalytic mechanism, the band gap energy of CdSe Nps is lower than the photon with energy. Hence CdSe photocatalyst absorbs the photons and creates the electron-holes pairs between the two energy levels. Doping the CdSe Nps either with metal nonmetal and rare earth metal can inhibit the recombination of electron hole pairs due to reduction of size and band gap in an effective way than the pure Nps. 
Transition elements play an important role in the doping mechanism like $\mathrm{Zr}$, Ti, $\mathrm{Zn}$, Mo, etc have been added to the CdSe catalyst to enhance the activity of host materials $[13,14]$. Among these metals, we have analyzed the photocatalytic activity of Ti-doped CdSe nanoparticles under solar irradiation with different parameters such as initial concentration, dosage and illumination time.

\section{MATERIAL AND METHOD}

\subsection{Chemicals and Reagents}

The analytical grade of chemical were used without further purification, cadmium chloride (99.9\%), sodium selenite (99.9) titanium oxide and TGA were purchased from Lobo Chemie. Deionised water was used throughout the experiment.

\subsection{Synthesis of Ti-doped CdSe Nps}

Ti-doped CdSe Nps were synthesized by using wet chemical method. In this synthesis, $0.1 \mathrm{M}$ cadmium chloride was mixed with $0.3 \mathrm{M}$ TGA and the $\mathrm{pH}$ was set in the range of 7-8. To the above solution, $50 \mathrm{ml}$ of $0.1 \mathrm{M}$ sodium selenite in the presence of TGA was added under vigorous stirring to give a reddish orange color solution. The freshly prepared $0.04 \mathrm{M}$ concentration of titanium dioxide solution was added drop wise to the above mixture and maintained the $\mathrm{pH}$ level at 7-8 and obtained red colour solution. The precipitate was taken out by adding propanal, filtered, washed, and dried in hot air micro oven at $80^{\circ} \mathrm{C}$ for 2 hours. Red color product was obtained [15].

\subsection{Photodecolortion experiments}

Stock solution (1000 ppm) of MB dye was prepared. The desired concentration (5-50ppm) was diluted using the stock solution. Exactly $20 \mathrm{ml}$ of the dye solution of known initial concentration (Co) was taken in a separate glass beaker. Required amount of catalyst was exactly weight and then transferred to the reaction solution. This solution was stirred for 30min by using a magnetic stirrer. During this period, catalyst and dye solution was attaining equilibrium state. After stirring the reaction, solution was irradiated under sunlight. After definite period of irradiation, the solution was centrifuged immediately for separating the dispersive solid particle. The solution was analyzed by measuring the absorbance value using calorimeter. Colour changes of methylene blue dye was shown in fig. 1.

$$
\text { Percentage removal }(\% R)=100 * C_{i}-C_{f} / C_{i}
$$

Where, $\mathrm{C}_{\mathrm{i}}$ is initial concentration of dye (ppm), $\mathrm{C}_{\mathrm{f}}$ is final concentration of dye (ppm) at given time.

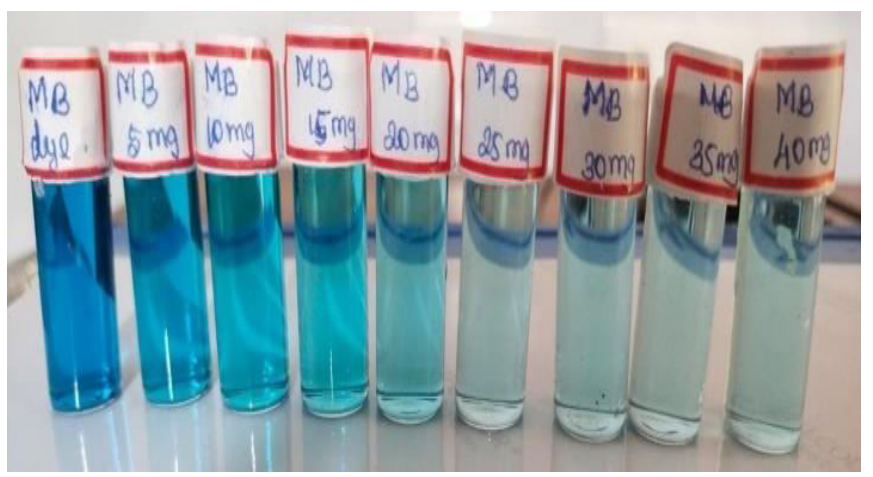

Fig. 1: Decoloration of Methylene Blue dye

\subsection{Characterization}

The synthesized sample was characterized by XRD, UV, FTIR, TGA, SEM, EDAX, TEM analysis. X-ray diffraction (XRD) pattern was recorded by using Bruker D8 advance powder $\mathrm{XRD}$ with $\mathrm{Cu}=\mathrm{K} \alpha(\lambda=1.5406 \AA)$ radiation. Uv-visible was analyzed with the help of AGILENT 5000, UV-Vis spectrophotometer in the spectral range between $200 \mathrm{~nm}$ to $700 \mathrm{~nm}$. The FTIR analysis was recorded on the Thermo Nicolet Avatar 370 in the range of wave number is $4000 \mathrm{~cm}^{-1}$ to $400 \mathrm{~cm}^{-1}$. The microstructure was recorded on the Jeol/JEM2100 with the point of resolution $0.23 \mathrm{~nm}$ and also recorded in the SAED pattern.

\section{RESULTS AND DISCUSSION}

\subsection{Crystallographic Analysis}

The average size and crystalline structure of undoped and Ti-doped CdSe nanoparticles were evaluated from the XRD pattern. Fig. 2 illustrates the XRD spectrum of undoped and Ti-doped CdSe nanoparticles. The peaks was obtained at around $25^{\circ}, 42^{\circ}$ and $48^{\circ}$, corresponds to the miller Indies of (111), (220) and (311) plane of cubic phase crystalline structure $[16,17]$ as given in table 1 which coincide with the standard JCPDS file no 09-0191 [18, 19]. After doping of $\mathrm{Ti}$ impurities, matching of crystalline structures of undoped nano materials couldn't be changed. The intensity and broadening of the preferred oriented (111) plane was higher than the undoped CdSe nanoparticles which confirms highly crystalline nature of prepared Ti-doped sample [20] and also higher the broadening of peaks, lower the size of synthesized samples [21]. The diffraction peak edges were shifted to the higher $2 \theta$ angle, which confirms the inclusion of Ti impurities into CdSe crystal lattice

The average grain size was estimated from Scherrer formula

$$
\mathrm{D}=\mathrm{K} \lambda / \beta \operatorname{Cos} \theta
$$


Where $\beta$ is FWHM, $\lambda$ is the wavelength of $\mathrm{X}$-Ray, $\theta$ is the angle of reflection, $\mathrm{K}$ is the constant and the particles size found to be $23 \mathrm{~nm}, 21 \mathrm{~nm}$ for CdSe and Ti-doped CdSe Nps. The reduction of particle size indicates the radius of $\mathrm{Ti}^{4+}(0.68 \AA)$ was lower than the $\mathrm{Cd}^{2+}(0.98 \AA)$ [22, 23]. Hence, Ti impurities could be easily substituted in the CdSe lattice site. Lattice constants value of undoped and Ti-doped CdSe Nps were almost similar. The dislocation density value of Ti-doped CdSe Nps was slightly higher than undoped CdSe Nps due to reduction of particle size. Lattice constant and dislocation density value of undoped and Ti-doped CdSe Nps was evaluated from the XRD spectrum given in table 1. Lattice constant value was almost similar to the pure CdSe Nps as well as dislocation density enhanced by the reduction of particles size.

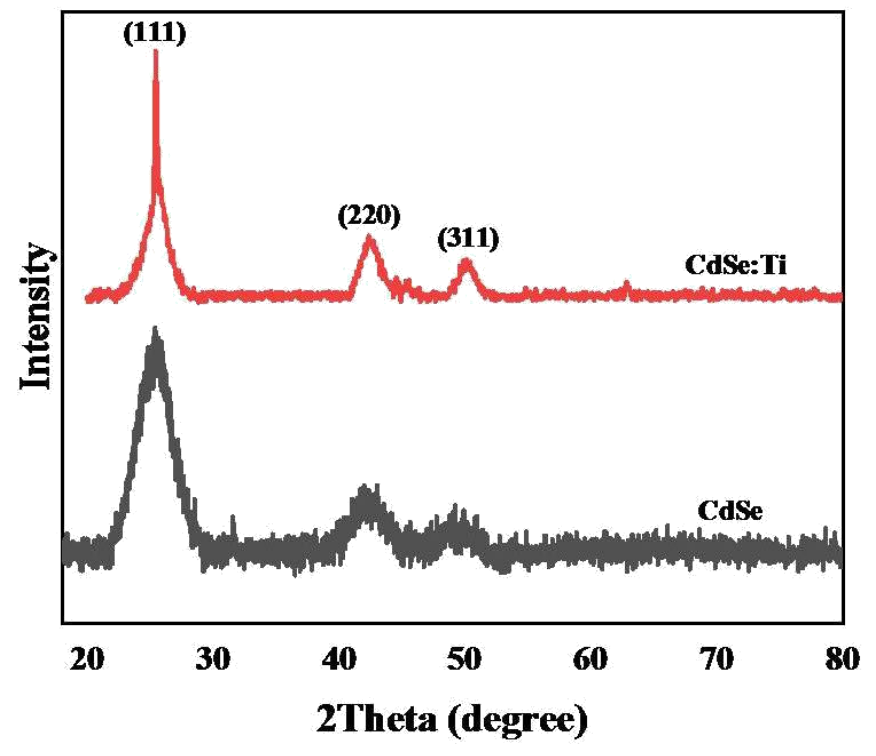

Fig. 2: XRD pattern of undoped and Ti-doped CdSe Nps

Table 1: XRD parameter of undoped and Tidoped CdSe Nps

\begin{tabular}{cccccc}
\hline Sample & $\begin{array}{c}\mathbf{2 \theta} \\
\text { Degree }\end{array}$ & Plane & $\begin{array}{c}\text { Size } \\
\mathbf{n m}\end{array}$ & $\begin{array}{c}\mathbf{a} \\
(\AA)\end{array}$ & $\begin{array}{c}\boldsymbol{\delta} \\
(\AA)\end{array}$ \\
\hline \multirow{2}{*}{$\mathrm{CdSe}$} & 25.3630 & 111 & & & \\
& 41.9739 & 220 & 23 & 6.1119 & 0.0189 \\
& 49.1746 & 311 & & & \\
\hline $\mathrm{CdSe}:$ & 25.4198 & 111 & & & \\
$\mathrm{Ti}(0.04 \mathrm{M})$ & 42.3337 & 220 & 21 & 6.1126 & 0.0226 \\
& 48.1830 & 311 & & & \\
\hline
\end{tabular}

\subsection{Optical Properties}

The UV-absorption spectrum is taken at room temperature at a fixed wavelength range of $200 \mathrm{~nm}$ to
$700 \mathrm{~nm}$. The absorption spectrum of undoped and Tidoped CdSe Nps was depicted in fig. 3. The absorption edge was observed at $305 \mathrm{~nm}$ and $274 \mathrm{~nm}$ for CdSe and Ti doping CdSe Nps. After doping Ti impurities absorption intensity was slightly different than undoped, this leads to the distortion of host materials. The absorption edge was transformed to the lower wavelength region than the bulk materials providing a blue shift according to quantum confinement effect [24-26].

Band Gap energy was evaluated from the Tauc plot [27, 28]. The extrapolating straight line portion $(\alpha h v)^{2}$ vs $h v$ along the $\mathrm{x}$-axis as illustrated in fig. 4. The band energy was found to be $3.37 \mathrm{ev}, 3.1 \mathrm{eV}$ for undoped and Tidoped CdSe Nps. The band gap energy decreases with the reduction of size due to quantum size effect $[29,30]$. Addition of $\mathrm{Ti}$ impurities in the host materials to generates higher lattice distortion in CdSe crystal lattice.

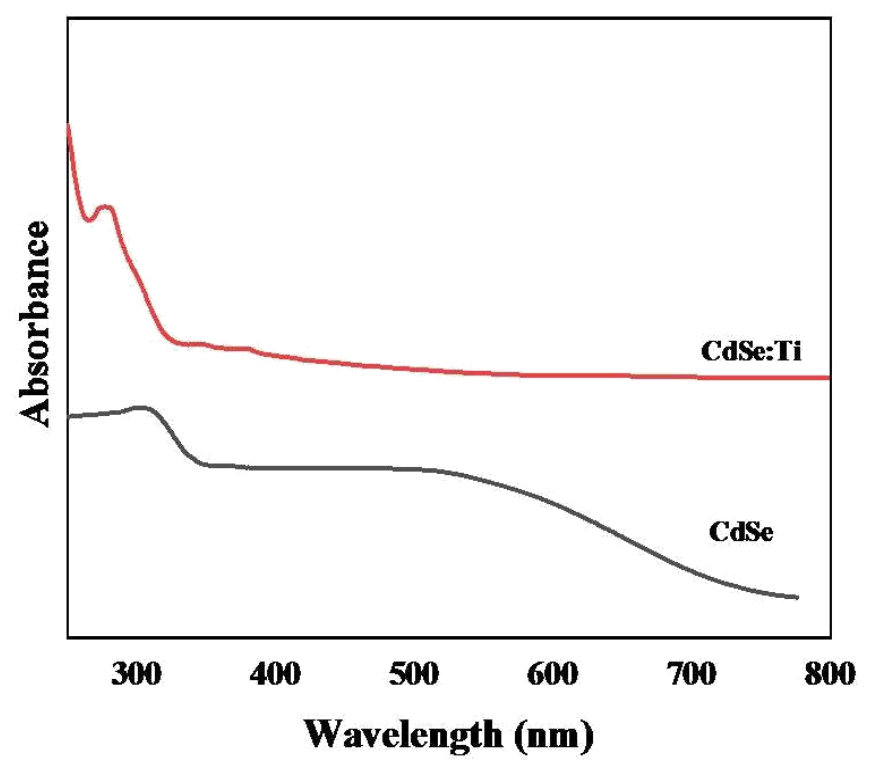

Fig. 3: UV-visible spectrum of undoped and Tidoped CdSe Nps

\subsection{Functional Group Analysis}

The synthesized sample functional group can be accessed from the FTIR spectrum. The $\mathrm{O}-\mathrm{H}$ stretching vibration of water molecules was observed at $3500 \mathrm{~cm}^{-1}$ as seen in fig. 5. The sharp peak at the range of $2362 \mathrm{~cm}^{-1}$ to $2354 \mathrm{~cm}^{-1}$ denotes the $\mathrm{C}-\mathrm{H}$ stretching vibration of the binding agent. The band obtained at $1726 \mathrm{~cm}^{-1}$ to 1730 $\mathrm{cm}^{-1}$ was assigned to be $\mathrm{C}-\mathrm{O}$ stretching band of carboxylic acid group. In addition, the FTIR spectrum was slightly shifted to a higher wavenumber region as it confirms the Ti inclusion in the host materials. The broad band at the range of $1558 \mathrm{~cm}^{-1}$ to $1543 \mathrm{~cm}^{-1}$ indicates the 
asymmetric vibration of $\mathrm{COO}$ - group due to the stabilizing agent that exists in the form of carboxylate salt. The small peak at $1110 \mathrm{~cm}^{-1}$ to $1072 \mathrm{~cm}^{-1}$ shows the $\mathrm{C}-\mathrm{H}$ bending vibration. The weak bands at below $1000 \mathrm{~cm}^{-1}$ to $400 \mathrm{~cm}^{-1}$ denotes the starching and vibration of Cd-Se band. The spectrum for the thiol group is not seen because it is completely adsorbed on the surface of metal Nps [15]. FTIR spectrum confirms the Ti-doped CdSe Nps capped with thioglycolic acid and its control the growth of nanoparticles.

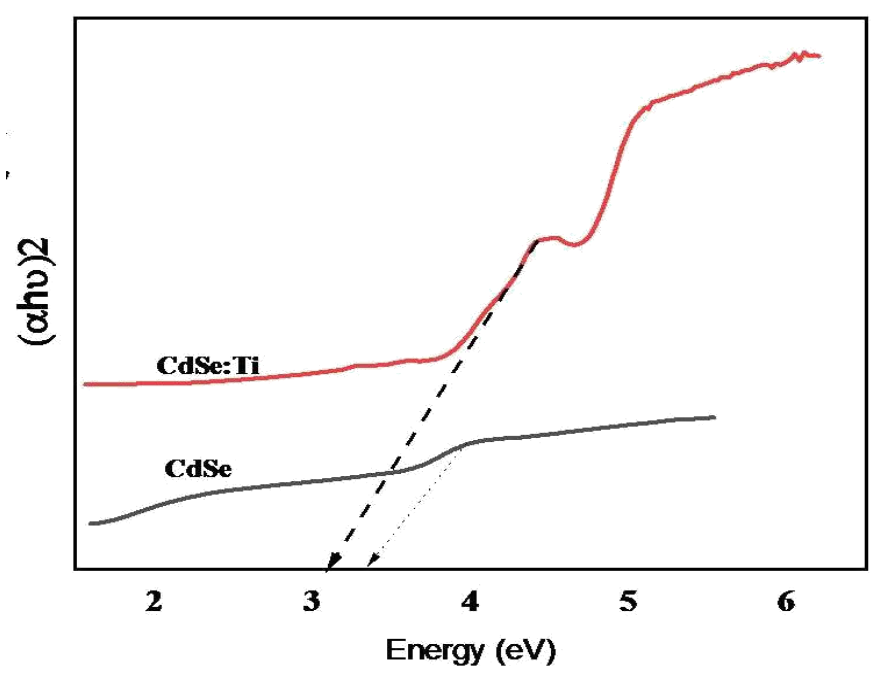

Fig. 4: Tauc plot of undoped and Ti-doped CdSe Nps

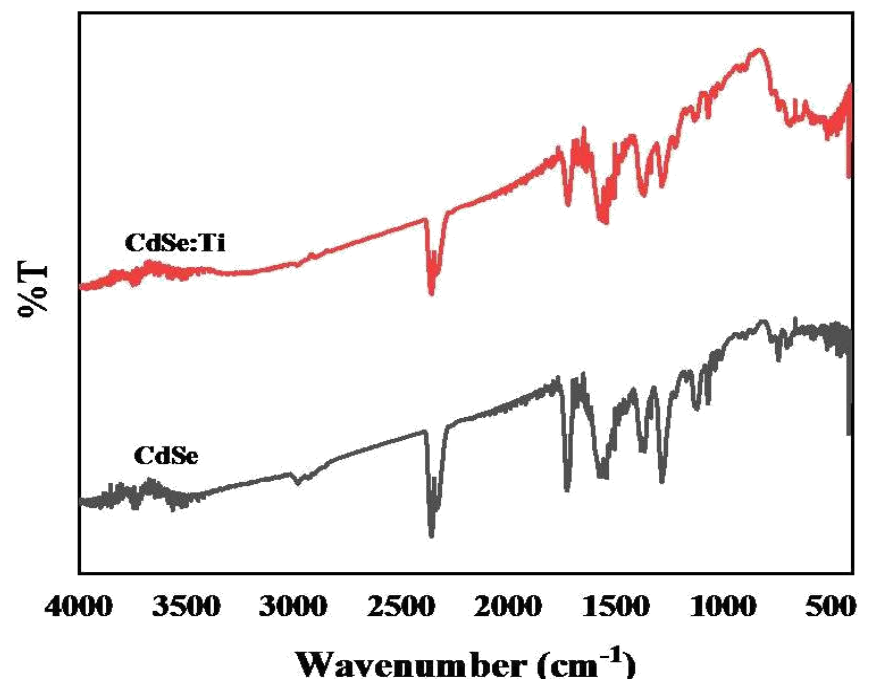

Fig. 5: FTIR spectrum of undoped and Ti-doped CdSe Nps

\subsection{Thermal Properties}

The stability of the preferred samples was confirmed by TGA analysis. The sample was heated at the range of room temperature to $750^{\circ} \mathrm{C}$, to get rid of water and organic residue. Fig. 6 shows the TGA spectrum of undoped and Ti-doped CdSe Nps. Two weight loss curves were obtained, the first drop was obtained between $80^{\circ} \mathrm{C}$ to $235^{\circ} \mathrm{C}$ mainly corresponds to desorption of water molecules and the steady weight loss was obtained at the range of $220^{\circ} \mathrm{C}$ to $432^{\circ} \mathrm{C}$ indicates the removal of organic residue from the synthesized. The temperature increased from $450^{\circ} \mathrm{C}$ to $750^{\circ} \mathrm{C}$, no significant weight loss was obtained. Finally, pure crystalline samples were obtained with complete desorption of water and organic residue. This spectrum confirms that the synthesized Ti-doped CdSe Nps were thermally stable.

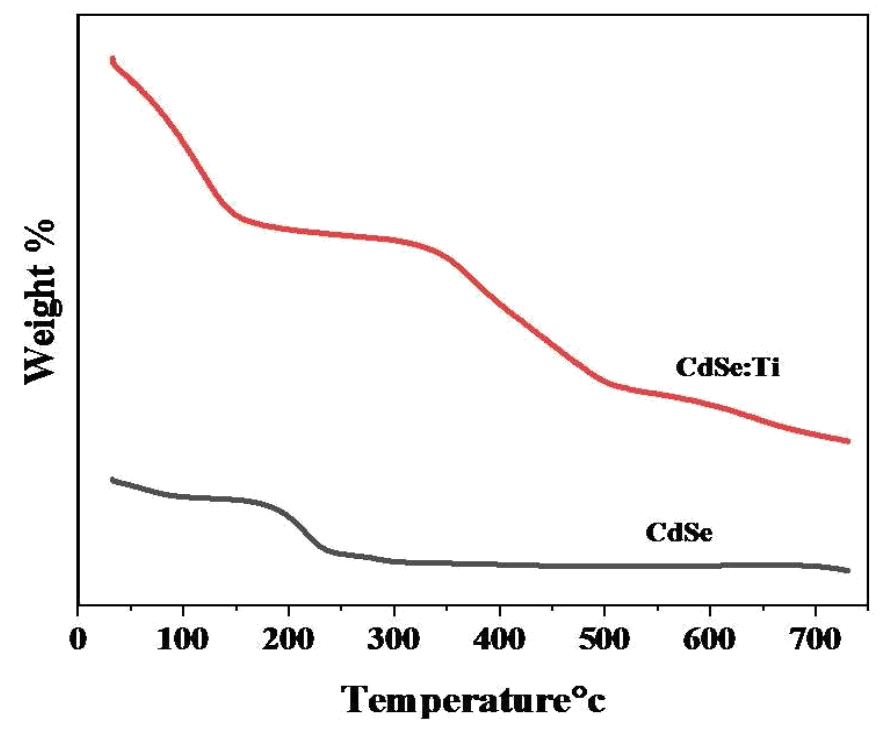

Fig. 6: TGA spectrum of undoped and Ti-doped CdSe nanoparticles

\subsection{Morphology Study \\ 3.5.1. SEM and EDAX Analysis}

Fig. 7 (A, B) shows the SEM micrographs of undoped and Ti-Doped CdSe Nps. The SEM images were highly agglomerated with irregular shape and size. Ti-doped CdSe Nps images shows the particles were interspersed on the surface. The grain size ranges from $310 \mathrm{~nm}$ to $370 \mathrm{~nm}, 145$ to $260 \mathrm{~nm}$ for undoped and Ti-doped CdSe Nps and confirms the reduction of particles size after doping of Ti impurities.

EDAX analysis is also performed in order to confirm the incorporation of Ti impurities in the CdSe matrix. Fig.7. (C, D) depicts the peak observed at $3.15 \mathrm{KeV}, 1.4 \mathrm{KeV}$ and $4.5 \mathrm{KeV}$ for $\mathrm{Cd}$, Se and $\mathrm{Ti}$ elements respectively. No other secondary element have been detected which indicates the $\mathrm{Ti}$ incorporated into the host materials. 

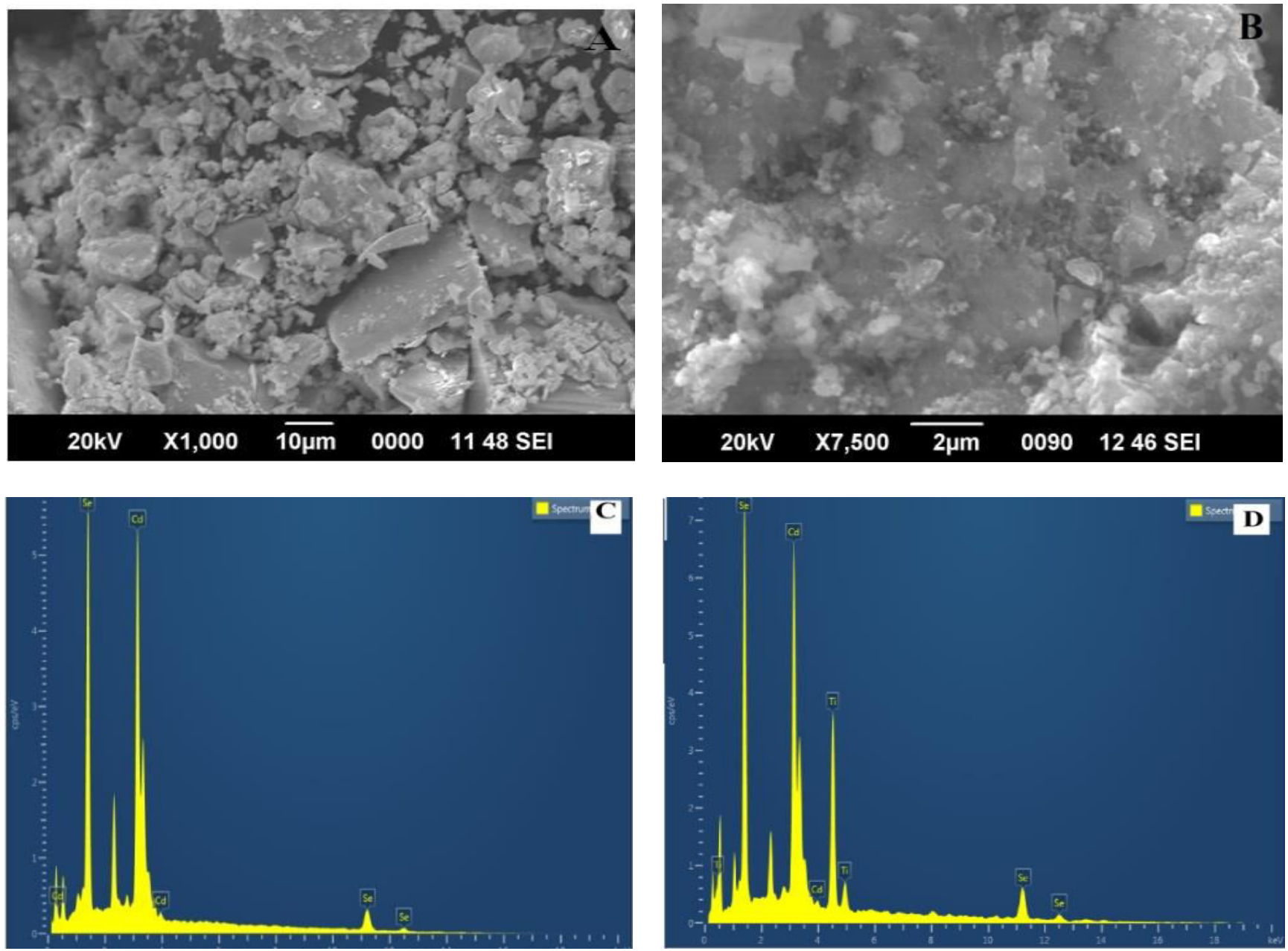

Fig. 7: SEM image of (A) CdSe and (B) Ti-doped CdSe Nps and (C, D) EDAX spectrum of CdSe and Tidoped CdSe Nps

\subsubsection{TEM and SAED Analysis}

The size and crystalline nature of Ti-doped CdSe Nps has been computed by using transition electron microscopy. TEM image of Ti-doped and undoped CdSe Nps depicted in fig. 8 (A, B). These entire images show prominent spherical in shape without being uniformly distributed and also TEM image of Ti-doped CdSe Nps show a large spherical shape along the radial direction.

Fig. 8 (C, D) shows the SAED pattern of Ti-doped CdSe Nps. It consists of three fringes, corresponding to miller indices (111), (220) and (311) planes respectively indicating the formation of cubic phase crystal structure [31, 32]. The SAED patterns of Ti-doped CdSe Nps contain dark white spots which denote the synthesized doped samples of highly crystalline nature. The lattice distance of undoped and Ti-doped CdSe Nps was 3.9A. Lattice space value of the doped sample was similar to the undoped CdSe Nps. The SAED pattern result was coincidence with the result of XRD diffraction pattern.

\subsection{Determination of Decoloration Efficiency}

3.6.1. Effect of Initial Concentration and Dosage of catalyst under Solar Illumination

The concentration of organic pollutant affects the degradation efficiency. The effect of initial concentration of methylene blue dye on degradation efficiency of synthesized photocatalyst was analyzed at different concentrations from 5-50ppm with constant dose and time at $20 \mathrm{mg} / 20 \mathrm{ml}$ and $45 \mathrm{~min}$ respectively. Fig. 10 (A) shows the degradation efficiency of $\mathrm{Ti}$ doped and undoped CdSe Nps towards MB dye. The initial concentration of $\mathrm{MB}$ dye increases with decreasing decoloraization efficiency from 41.6-12.3\% for CdSe Nps and 45.8-12.7\% for Ti-doped CdSe Nps. The reduction of degradation efficiency indicates the intensity of MB dye higher hence light could not easily 
penetrate. It may be caused due to the fact that absorption of light energy was insufficient to transport the $\mathrm{e}^{-}$from valence band to conduction band as well as the concentration increases, $\mathrm{OH}^{\cdot}$ radicals generation get

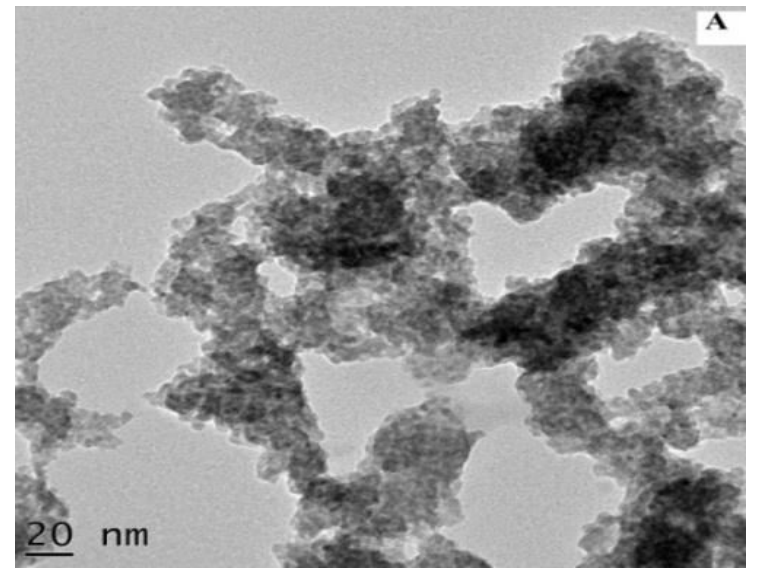

Fig. 8: TEM image of (A) CdSe and (B) Ti-doped CdSe Nps
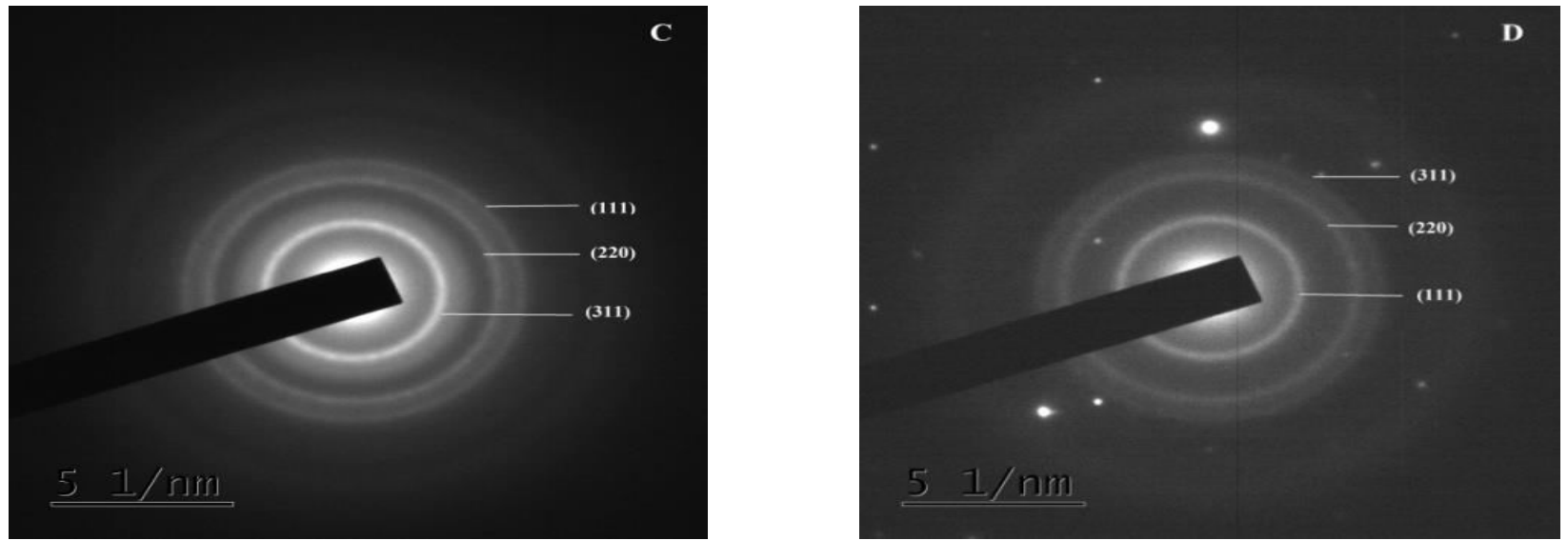

Fig. 9: SAED pattern of (C) CdSe and (D) CdSe:Ti Nps

The dose of photocatalyst is highly affecting the rate of degradation reaction. Optimum amount of catalyst was calculated from this experiment and leave the usage of excess photocatalyst. This experiment was carried out by different amount of catalysts from $5 \mathrm{mg}$ to $40 \mathrm{mg}$ and fixed the constant time and initial concentration at $45 \mathrm{~min}, 20 \mathrm{ppm}$. Dosage of catalyst was enhanced with increasing the percentage of degradation from 18.8 to $61 \%$ for Ti-doped CdSe Nps and 10.5 to $54 \%$ for pure CdSe Nps as depicted in fig. 10 (B). It may be due to the higher surface area and lower size of photocatalyst which enhanced driving force for transfer the electrons from $\mathrm{Ti}$ to $\mathrm{CdSe}$ and the dopant to avoid the recombination of electron and holes recombination. Percentage of degradation was higher in Ti-doped CdSe Nps due to the presence of more number of active site and hydroxyl radical [36-37]. decreased. In addition active site of photocatalyst was blocked by the adsorption of more number of dye molecules [33-35].

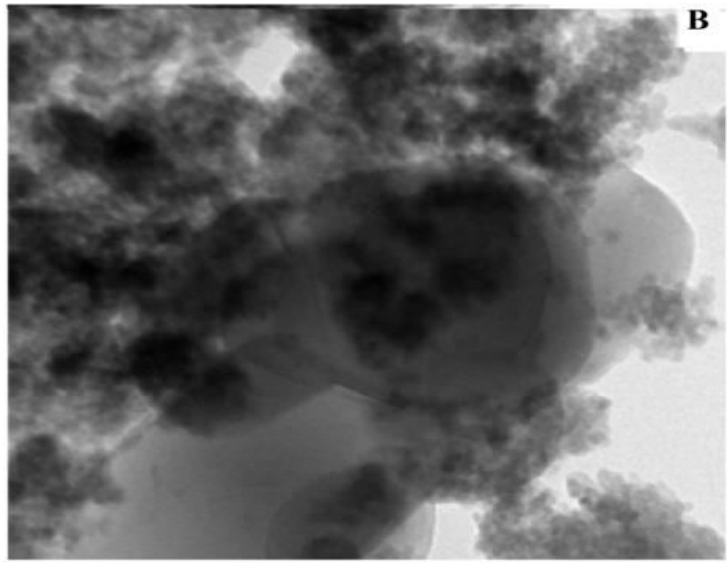




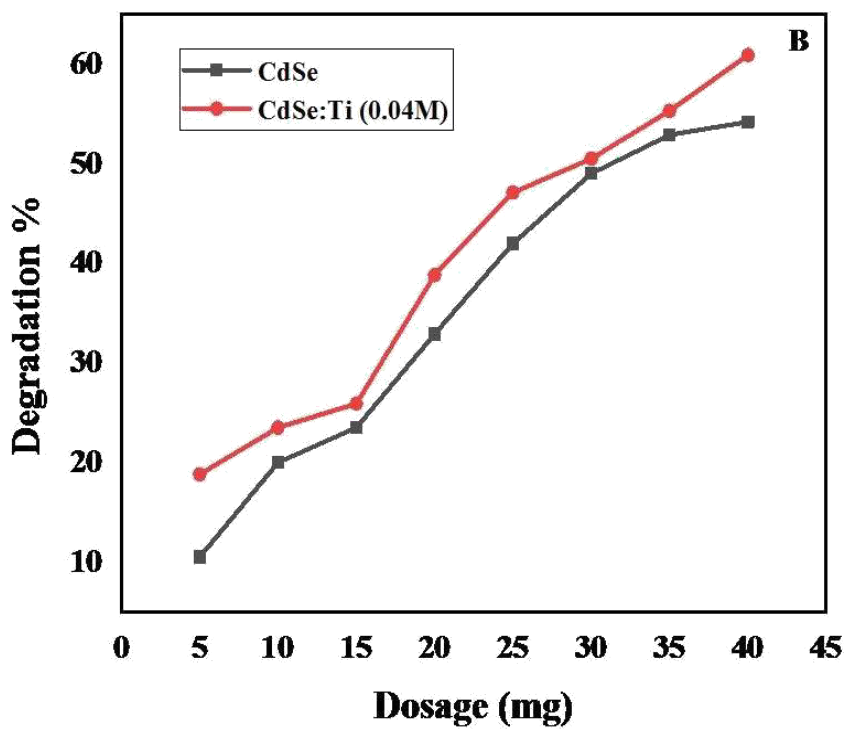

Fig. 10: (A) Effect of initial dye concentration and (B) Effect of dose of the catalyst on the photodegradation of MB dye under solar irradiation

\subsubsection{Effect of Solar light Illumination Time}

The irradiation time is the crucial parameter for degradation of MB dye. This process was carried out at the constant dosage and initial concentration with different contact time from $5 \mathrm{~min}$ to $70 \mathrm{~min}$ respectively. Decoloraization efficiency of MB dye with undoped and Ti-doped CdSe Nps under irradiation of solar light increases with increasing contact time as shown in fig. 11.

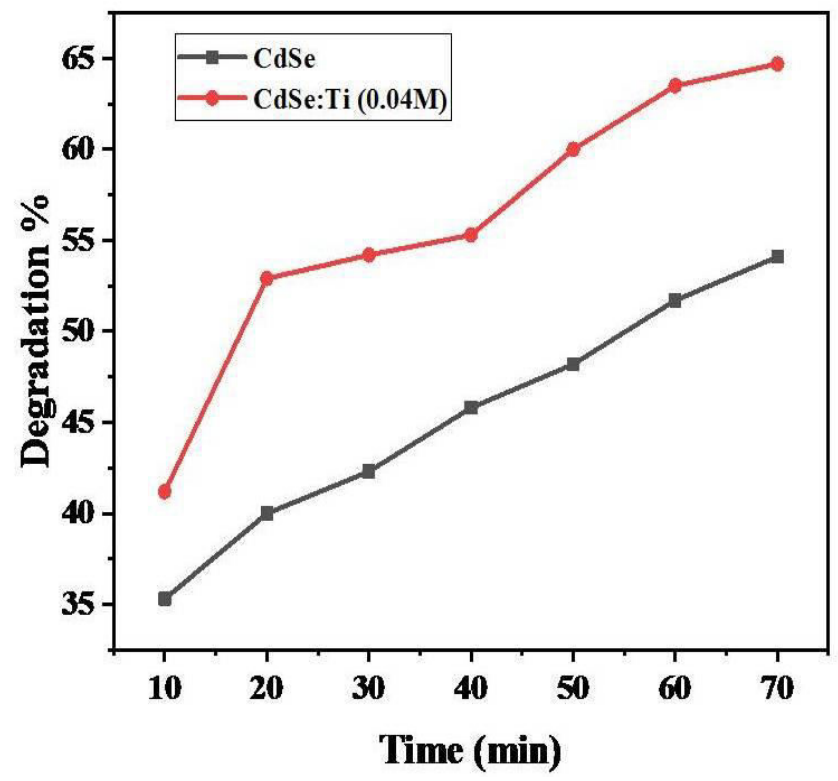

Fig. 11: Effect of contact time on the photodegradation of MB dye under solar irradiation
Degradation efficiency of undoped CdSe Nps was 35.3 to $54.2 \%$ and 41.2 to $64.7 \%$ for Ti-doped CdSe Nps. Ti doped photocatalyst highly removed the colour of MB dye than the undoped catalyst because the size, shape and band gap affect the activity of photocatalyst. Low concentration of $\mathrm{Ti}$ impurities given higher degradation efficiency hence increases the concentration of dopant to enhance the degradation efficiency respectively.

\section{CONCLUSION}

Undoped and Ti- doped CdSe Nps were synthesized via wet chemical method. The particles size of Ti-doped CdSe Nps was lower than the mere CdSe and confirmed by XRD analysis. The optical properties reveal the blue shift than the bulk materials and bandgap energy was enhanced from $3.37 \mathrm{eV}$ to $4.6 \mathrm{eV}$. TGA spectrum confirms the weight loss obtained at lower temperature range and the synthesized samples are thermally stable. Photocatalytic activity was found to decrease with increasing initial concentration and increase with increasing the dose of the catalyst and irradiation time. Ti-CdSe nanoparticles show higher decoloraization efficiency towards the MB dye under solar light illumination. Low electron-hole pair recombination with increased efficiency was observed under solar light due to the strong absorption by the photocatalyst under solar light.

\section{Conflict of interest}

None declared

\section{REFERENCES}

1. Tan X, Fang X, Tan L, Liu H, et al. Env. Sci. Nano Tech., 2018; 5:1140-1149.

2. Wu J, Chen, K, Ten X, Ming F, et al. J. Chem. Eng., 2018; 349:481-490.

3. Zhang Y, Zhu M, Zhang S, Cai Y, et al. Appl. Cata. B: Environ., 2020; 279: 119390-119397.

4. Vaseashta A, Vaclavikova M, Vaseastha S, Gallios S, et al. J. Mol., 2018; 23:1760-1766.

5. Park Y, Lee S, Kang SO, Cai Y, et al. Chem. Com., 2010; 46:2477-2479.

6. Linsebiger AL, Lu GR, Yates JT. Chem. Rev., 1995; 95:735-758.

7. Kumar A, Pandey G. Mat. Sci. Eng. Int. J, 2017; 1:106-114.

8. Lin F, Shao B, Li Z, Zhang. J.App. Catal. B: Environ., 2017; 218:480-487. 
9. Tratnyek PG, Johnson RL. Nano Today, 2006; 1:4448.

10. Wang H, Zhang I, Chen Z, Hu J, et al. Chem. Soc. Rev., 2014; 8:5234-5244.

11. Schneider J, Matsuoka M, Takeuchi M, Zhang J, Horiuchi Y, et al. Chem. Rev., 2014; 114:9919. 9986.

12. Lo S, Lin C, Wu C, Hsie P. J. Hazar. Mater B, 2004; 114:183-190.

13. Suo Z, Dai J, Gao S, Gao H. Res. Phys, 2020; 17:103058.

14. Rajesh Kumar B. Chalco Lett., 2015; 12(4):181-190.

15. Anne kausalya V, Joseph, Krishnakumar S. Appl. Chem., 2013; 55A:13036-13038.

16. Gao Y, Yin PG. Nanomat. Nanotech, 2017; 20:1-6.

17. Dlamini NN, RajasekharPullabhotla VSR, Revaprasadu N. Mater Lett., 2011; 65:1283-1286.

18. Kristl M, Ban I, Danc A, Drofenik M. Ultrasonic.Sono Chem., 2010; 17:916-922.

19. Chen ML, Oh WC. Nano. Res. Lett., 2011; 6:398405.

20. Wang C, Shi H, Li Y. Appl. Surface Sci.,2012; 258:4328-4333.

21. Kumar S, Sunil K, Verma NK. Appl. Nanosci., 2012; 2:127-131.

22. Gnanasekaran L, Hemamalini R, Saravanan R, Ravichandran K, et al. J.Molec. Liquids, 2016; 223:652-659.

23. Hamizi NA, Johan MR, Ghazali YA, Wahab ZZ, et al. Processes, 2019; 4:7639-7647.
24. Shahi AK, Pandey BK, Singh BP, Gopal R. Nanosci. Nanotech. 2016; 7:35010-35016.

25. Rodríguez-Mas F, Carlos Ferrer J, Luis Alonso J, Valiente D, Fernández de Ávila S. Crystals, 2020; 10:226-234.

26. A Book: Woggon U. Springer, Tracts in Morden Physics, 1997. p. 136.

27. Book: Tauc J. Amorphous and Liquids Semiconductors, Plenum Press. New York: 1974

28. Mahmoud WE, Al Amri AM, Yaghmour SJ. Pot. Mater, 2012; 34(7):1082-1086.

29. Singh V, Priyanka Vilas More P, Hemmer E, et al. Mate. Adv., 2021; 51 (2):1204.

30. Kumar P, Singh J, Kumar Pandey M, Jeyanthi CE, et al. Mater. Res. Bullet., 2014; 49:144-150.

31. Yadav K, Dwivedi Y, Jaggi N. J. Lumininsci., 2015; 158:181-187.

32. Mukta V, Limaye B, Shashi Singh B, Sadgopal K, et al. Mater. Res. Bullet., 2009; 44:339-344.

33. Zhao D, Sheng G, Chen C, Wang X. Appl. Catal. B. Environ., 2012; 111:303-308.

34. Wang WS, Wang DH, Qu WG, Lu LQ, et al. J. Phys. Chem. C, 2012; 116:19893- 19901.

35. Muthirulan P, Nirmala Devi C, MeenakshiSundaram M. Ceram. Inter., 2014; 40:5945-5957.

36. Khalid NR, Ahmed E, Hong Z, Zhang Y, Ullah Ahmed M. Ceram. Inter., 2013; 39:3569-70 3575.

37. Liu H, Dong X, Duan C, Su X, Zhu Z. Ceram. Inter., 2013; 8789-8795. 